\title{
REVIEW ON SMART TRAFFIC MANAGEMENT SYSTEM FOR AMBULANCE
}

\author{
Deepika Sarpal
}

Electronics and Communication Engineering, Vellore Institute of Technology, Vellore, India

\section{Yatharth Asthana}

Electronics and Communication Engineering, Vellore Institute of Technology, Vellore, India

\section{Dr Malaya Kumar Hota}

Professor, Department of Communication Engineering, School of Electronics Engineering, Vellore Institute of Technology, Vellore, India

\begin{abstract}
Rising traffic clog is an unpreventable condition in enormous and developing metropolitan zones all over India. An increased volume of vehicles not only increases the chances for them being involved in accidents. According to a report published in Times of India, 146,133 people were killed in road accidents in India in the year 2016. Unfortunately, about 30\% of deaths are caused due to delayed ambulance. Another data shows that more than 50\% of heart attack cases reach hospital late. Everybody detests traffic clog, and it continues deteriorating, despite endeavoured cures. In this manner, emergency vehicles stuck in a road turned parking lot and deferred in arriving at their goal can cause loss of property and significant lives. This paper provides the knowledge of all kind of techniques used till now to make traffic management system for emergency vehicle better and make it easier for people to reach on time to the hospital. Explain ways to get the best route while maintaining a cloud using IoT based system and various algorithms to various ways to detect ambulance using image processing and fuzzy logic.
\end{abstract}

Key words: IoT, Embedded Systems, Machine Learning, Artificial Intelligence, Image Processing, Neural Networks.

Cite this Article: Deepika Sarpal, Yatharth Asthana and Dr Malaya Kumar Hota, Review on Smart Traffic Management System for Ambulance. International Journal of Electrical Engineering and Technology, 11(6), 2020, pp. 1-9.

http://iaeme.com/Home/issue/IJEET?Volume $=11 \&$ Issue $=6$ 


\section{INTRODUCTION}

In India, 33\% and $99 \%$ of people die in road accidents and heart attacks respectively because of the late arrival of Ambulance or delay in reaching hospitals due to traffic jams. According to the National Crime Records Bureau, nearly 24,012 people die each day due to a delay in getting medical assistance. The first hour after an emergency is the critical hour for medical aid to the patient for saving a life.

What is required to improve ambulance arrival on time to hospitals as well as on-site? Major metro political cities like Mumbai, Delhi, Bangalore, Chennai, Hyderabad, Kolkata, Jaipur, Lucknow and much more advancing cities face the problem of traffic jams in leading hours. For reducing congestion, it requires to observe the intensity of vehicles on one side rather than going for a timing-based system. Necessarily for emergency vehicles, so many IoT and Artificial Intelligence-based systems can be utilized to give Ambulance priority rather than any other vehicle as life is very precious.

This paper presents different methodologies gist to deal with emergency vehicles in rush hour gridlock. IoT can be used to track the vehicles and detect the exact location with an asset of Artificial Intelligence. Embedded system has also been proved to be very beneficial and has the potential to minimize the waiting time of emergency vehicles. The system can be improvised in combination with machine learning algorithms and neural networks. The information on recognizing the emergency vehicle is sent to the traffic system through the RF transmitter and receiver or ZigBee, for adequately controlling the traffic light until the emergency vehicle experiences. The condition of Indian traffic makes it hard for the emergency vehicle to reach the destination on time. So to decrease the response time of ambulance, a precise traffic control system is used, which turns the traffic signal green for an emergency vehicle.

\section{LITERATURE REVIEW}

India is one of the fastest-growing vehicle markets with the ranking of the fourth largest car manufacturer in 2018 across the world, expanding at double-digit annual rates. However, the increasing number of vehicles is not only increasing the ambulance response time. Still, it is also increasing the number of accidents happening every year with traffic congestion as the most significant problem with less developed road structure across the country.

Traffic makes a demand for a system to overcome delay issue and researchers started finding the solutions. Therefore, by the end of 2004 [1], a real-time response system was introduced using dynamic shortest path algorithm which makes use of first-come, first-serve basis. Allot patients to the nearest Ambulance(s) based on the severity of the patient or accident and tell the path with least traffic to the driver.

Some sensor-based systems came into use but were not having much effect as their results were not accurate then RFID gets introduced [2][3][4][5][6][7][8][9][10][11][12], having vehicle identification number, send to the system that computes the density. A decision made if more frequency of vehicles is detected then the threshold, the signal is made green that side by giving buffer time of some seconds on the ongoing direction. RFID used for emergency detection [3].In this, after Ambulance reaches near traffic lights; it broadcasts a priority intersection control request and then waits for the grant based on priority setting. After the Ambulance crosses the intersection, it transmits a clear message for the next operation. In [11], RFID and GPS based system is used to detect the ambulance presence and microcontroller is used to control traffic lights.

Fuzzy logic-based system [5] involves reducing the response time of ambulance by changing traffic light or speed limit or lane clearance or permission to use reserved lanes and 
re-routing. Two conditions are defined 1.Emergency Level (EL) 2.Congestion Level (CL) between 0 to 1 which acts as a decision control and tells the fuzzy logic controller about a correct emergency plan to be implemented.

RF technology is efficiently in use as a wireless communication medium $[12][13][14][15][16]$. The [13]paper states that the timing-based traffic control is in use until the emergency vehicle came near to the intersection point of the traffic light. The driver is having a remote with four buttons, when pressed sends the coded signal to RF receiver making that signal green for ambulance clearance. In [15] uses RF Tx in the ambulance when came near $100 \mathrm{~m}$ of traffic junction with four buttons and the control unit have Arduino and $\mathrm{RF} \mathrm{Rx}$ to make a particular signal green for some seconds.[16] uses RF present in an ambulance for transmitting emergency presence near the junction to the microcontroller which gives information to traffic lights.

An adaptive Traffic Management System algorithm is found [17]. It helps in characterizing ambulances in three priorities and provides different policies are made routing vehicle accordingly by live tracking using GPS. Input with CCTVs and sensors giving to Local traffic controller which offers information to Traffic Management Controller (TMS). V2V and V2I communication take place for smooth working, which involves broadcasting SMS to all non-emergency vehicles about the severity of the patient and the way needed. Sublocations to have a different local traffic controller that gives their data regularly to the TMS.

Embedded systems became popular with some more improvement and flexibility than previous models [4][6][7][14][18][19][20][21][22]. [4] Uses RFID to detect the density of the vehicles and change signals accordingly. PIC microcontroller is the brain of the system. Both in traffic controller and vehicle ZigBee and buzzer are installed. Whenever an ambulance come in range, the buzzer gets on, and the light turns green. Stolen vehicle detection, implemented using RFID tag when matches the stolen vehicle, the traffic of the lane paused for action, and a message is sent to police using the GSM module so that local police can take action. In [7], RFID tags are used for ambulance detection with Arm7 microcontroller as a brain. Hybrid communication is used to communicate from vehicle to gateway and vice-versa, which have less cost and more effectiveness comparing to V2V and V2I. [18] Uses IR sensors for detecting the density of vehicles and Wi-Fi is used to transfer the location of Ambulance to the traffic light system using GPS and providing a way to Ambulance until in range. In [22], the programmable logic controller is used as the brain to control the signals and take input from sensors which detects the presence of ambulance and change signals accordingly.

Distance-based emergency vehicle dispatching algorithm [19] emphasizes on vehicle counting and Sensors sense the emergency vehicle presence in the region of interest. If there are more than one emergency vehicles, then controller checks if they are at the same distance from intersection or not. If the ambulance is at the same distance then chosen randomly or if not then arranged in ascending order of reach as a priority. Green light duration is calculated based on values measured. Then speed and preference of the vehicle are verified, and nonemergency vehicles are counted, and value is sent to the next traffic intersection.

In [20] ambulance unit and data centre (software) make traffic management more advanced as it involves GSM and GPS installed in ambulance connected with Arduino microcontroller. GPS signals are sent continuously to the data centre web page, and the database table is maintained. The patient address is feed on the web page, and the nearest ambulance is searched, and then the route is sent to the nearest ambulance. If there are traffic interaction in the way, then data is sent to the traffic controller to make it green for an emergency.

Video Processing is a kind of Image Processing which allows live tracking of the realtime situation using algorithms[10][19][23][24][25][26][27][28][29][30].[23] Implements 
video processing as basic technique focusing on calculating the density of all four lanes, considering vehicle density according to the size of the vehicle as it will take more space and time to pass giving accurate results. [24] Uses a blob detection technique for counting the vehicle on a particular side of the junction. OCR does number plate extraction if a vehicle breaks the traffic, which is detected using an IR sensor. An ambulance is identified using MATLAB, and the particular signal turns green for the ambulance to pass. Displays warning messages according to the situation. [25] uses a web camera to take snapshots of the current traffic. Then MATLAB is used to count vehicles on the road and controls traffic lights using Arduino microcontroller based on density. [26] Traffic density is found using the combination of gradient magnitude and direct subtraction method. Pixel density tells if it is an emergency vehicle or not by converting RGB to HSV to a binary image.[27] Image processing using blob detection is used to count the number of vehicles present in the lane. Ambulance detection is done using a Bluetooth module connected to Arduino and a phone. When the ambulance reaches near the junction, it sends command using phone, making the change of signal. Rest density-based system is followed using Arduino and threshold set vehicle counts.[29] uses the approach of vehicle counting and emergency vehicle type detection using image processing and sensors data .real time data is then put to the analytic engine, which is analyzed using mobile agents at STMS system. When the vehicle frequency is more than the threshold, then a message is broadcasted to divert other vehicles to different lanes and when the clear message is transmitted telling normal density. In [30] YOLO-V3 used for detecting vehicles from the image given from video and the detection is done after training the model from the given COCO data set.

In [31] system is using Geographic Information System and Global Positioning System (GIS-GPS) for live tracking using GPS mobile and informing the registered user about traffic and accident-prone areas when asked from a source to destination. An application, used for interaction with a database and server for smooth working.

Dheeraj Dang, Jitin Tanwar, and Sarfaraz Masood[32] proposed a priority-based algorithm which focused on calculating priority value of all four sides and taking the decision to give way to an ambulance if the value is greater than zero. Suppose priority value is equal to zero than traffic system runs usually.

ZigBee technology[4][6][21][33][34] came with more range to control traffic light.[6] contains two units lane unit and ambulance unit. RFID reader detects emergency vehicles and lane unit informs vehicle presence to ambulance unit using ZigBee. Then vehicle information is checked, and particular light turns green, and after ambulance clearance, it turns red again.[34] uses sound sensors to find the ambulance frequency and predict its presence. The sound sensors communicate to Arduino using ZigBee module. Then accordingly, Arduino make changes in the traffic light.

IR sensors [18][21][24] detects traffic density based on its fundamental principle of recognizing object present at a particular distance.[21] uses IR sensors with PIC microcontroller and ZigBee. Four push buttons based portable controller is present with control of all sides of the traffic light. When 'A' button is pressed traffic light of particular side turns green till 'A' button presses for the second time, which returns traffic controller to normal working.

Kumar, G.H. and Ramesh, G.P[35] proposes a unique method of providing preference to an ambulance at the railway junctions with the live tracking of the train using GPS and giving SMS to the user using GSM. Motors are controlled using Arduino connected to the phone via Bluetooth. Spikes shafts are in a Z shape to reduce causality, and the gatekeepers are replaced with automatic motor spikes. 
IoT is a combination of Embedded Systems Technology, Network Technology and Information Technology [8][9][10][12][33][36][37][38][39][40][41][42][43][44][45][46][51] [52]. In [8], the mobile application is used to register, block or to show emergency/nonemergency. The phone GPS is used to track the nearest traffic post and after the RFID tag reads the ambulance give it to the microcontroller which conveys it to cloud and if an ambulance is in emergency mode the signal turns green. In [9], Arduino with RFID is used to detect density and emergency vehicle presence. The traffic light system is controlled according to the density of vehicles. The web server informs Arduino about the incoming ambulance, and the RFID information is matched. If matches then the way is given to the vehicle. [10] involves a camera, RFID, ultrasonic and flame sensors. Camera processed video undergoes blob detection algorithm, and then density calculation is done on server. Sensors input are given to microcontroller, which performs density calculation at the node. Both are added, and if the density is high, then some extra time is added based on the vehicles velocity and time it will take. Nextly, if an emergency vehicle is detected, then rest functions stops and make particular lane green for the vehicle to pass. Fire can also be detected and can be informed to the authority. In [12] uses RFID to detect ambulance and to detect vehicle density, and the data is transmitted to Arduino using RF technology. Then Arduino takes action and makes a path for an ambulance at the junction. In [33], three central systems are introduced.

The first system called intersection controller finds emergency vehicle presence using the system installed at traffic lights and density of vehicles present on a particular road Then intersection controller adjust traffic lights based on traffic detected on the road. The second system present at each path uses sensors to detect the presence of the vehicle and sends data to the controller using ZigBee. The third system is installed in the ambulance to provide GPS coordinates to the controller, which then decides to give way to the ambulance at the junction. In [36], different sensors are installed in the ambulance like ECG, heart and pulse rate sensor for getting information about the criticality of a patient. The data, along with traffic signal data, will be sent to hospital authority through the cloud. When the ambulance comes near to $100 \mathrm{~m}$, the signal will be made green through GPRS. In [37] and mobile application is integrated with the cloud which tracks the location of ambulance using phone GPS and sends it to server. When ambulance approaches a junction, the mobile sends information to the microcontroller to change the signal to green.

[38] introduces a new blue light which makes it much different from others. So that nonemergency vehicle knows the cause of the light change. Uses Raspberry Pi to create a server and to connect it to the traffic light. The mobile application uses phone GPS for live tracking of Ambulance and sends data to the server. Rpi takes charge after this for opening ways for an ambulance. In [40], MQTT cloud server is used with GSM to send messages and receive messages by Arduino and mobile application. Phone GPS is used to know the current location of an emergency vehicle and give way in + traffic junction. In [41], the GPS module is used to track the location of Ambulance and sends data to the cloud(ThingSpeak). Cloud interacts with NodeMCU using inbuilt Wi-Fi for opening traffic light when an ambulance approaches junction and buzzer is on which indicates emergency in a vehicle.[42] uses a different algorithm FOG. Fog node is made centrally available and installed on raspberry pi. The driver sets the destination, and the doctor inputs the severity of the case in the mobile app. GPS is used in an ambulance to provide route and live to track it. If more than one ambulance is there, then priority is given based on the criticality of patients. [43] focuses on the idea of using smartphones GPS location of both general vehicles and transmitting to server and ambulances. Whenever ambulance is near to public vehicle with about $500 \mathrm{~m}$ difference, then the general vehicle gets siren sound to clear the way for the ambulance.[44] focuses on MQTT broker and GPS of mobile to be in use and the STMS database is maintained of the 
location. The route is given to ambulance from source to destination through the mobile app, and Arduino controller is informed to clear traffic of that side before ambulance arrival to that point as database have information of current location and all route traffic lights. In [45] GPS is used for live tracking using an Android app which deals with asking driver the criticality of the case and ambulance destination and then the data is communicated to cloud which informs the upcoming signal about the ambulance and giving way condition.[46] comprises of raspberry pi in RSU and a NodeMCU in the ambulance. RSU and communicate wirelessly through Wi-Fi about priority and arrival of the ambulance and the ultrasonic sensor is responsible for calculating the density of the lane and ambulance presence.

In [47], density is derived from the data collected by the sensors after sending it to Raspberry pi, which sends data to the server, which is saving data in the cloud using MQTT protocol. It is a web-based system so users can be increased and getting traffic and alternative route information.

[48] Introduces CGA-PSO approach, which helps in re-routing ambulance or any emergency vehicle with velocity required and route information so that they can avoid congestion areas and hazardous zone. It proves that PSO is better than Dijkstra algorithm.

Convolutional neural network $(\mathrm{CNN})$ a deep learning neural networks deal with data arrays like images and have many layers between the input and output layer found using convolution.[49][50]. In [49] camera is used for providing image input from the environment and uses a multi-task CNN approach to detect vehicle as ambulance using the stochastic gradient-based solver with momentum. In [50] Ambulance is detected using Deep learning algorithm for copying fully and replacing human by introducing self-driving and making accidents to zero and giving way to the ambulance as its self-driving by computers so no breaking of rules.

\section{CONCLUSION AND FUTURE SCOPE}

Combination of IoT, AI and machine learning can lead to the advancement of the developing country like India as the health infrastructure will be improved, and people suffering will become less. In India, people suffer due to no ambulance availability or traffic jams which leads to an increase in waiting time and loss of a golden hour. But after building the infrastructure that connects everything to cloud, it becomes effortless to save lives and reduce jams. It becomes cost-effective if used in the whole city and connected to the control room and by giving access to the traffic police to do everything in an emergency so the very delicate case can also be handled.

\section{REFERENCES}

[1] Haghani, Ali, Qiang Tian, and Huijun Hu. "Simulation model for real-time emergency vehicle dispatching and routing." Transportation Research Record 1882.1 (2004): 176-183.

[2] Chattaraj, Anuran, Saumya Bansal, and Anirudhha Chandra. "An intelligent traffic control system using RFID." IEEE potentials 28.3 (2009): 40-43.

[3] Sharma, Suresh, et al. "Traffic light priority control for emergency vehicle using RFID." Int. J. Innov. Eng. Technol 2.2 (2013): 363-366.

[4] Sundar, Rajeshwari, Santhoshs Hebbar, and Varaprasad Golla. "Implementing intelligent traffic control system for congestion control, ambulance clearance, and stolen vehicle detection." IEEE Sensors Journal 15.2 (2014): 1109-1113.

[5] Djahel, Soufiene, et al. "Reducing emergency services response time in smart cities: An advanced adaptive and fuzzy approach." 2015 IEEE First International Smart Cities Conference (ISC2). IEEE, 2015. 
[6] Tiwari, Chandan, et al. "Automatic lane clearance system for emergency vehicles." Int. J. Inn. Res. Sci. Eng. Tech. 5.1 (2016): 392-396.

[7] Reddy, Benjaram Madhusudhan, Kiran Kumar Anumandla, and Vikas Kumar Tiwari. "Optimization of smart vehicle ad hoc network (SVANET) communication for traffic related issues with a security." 2017 International Conference on Computer Communication and Informatics (ICCCI). IEEE, 2017.

[8] Saradha, B. Janani, G. Vijayshri, and T. Subha. "Intelligent traffic signal control system for ambulance using RFID and cloud." 2017 2nd International Conference on Computing and Communications Technologies (ICCCT). IEEE, 2017.

[9] Naik, Tejas, et al. "RFID-based smart traffic control framework for emergency vehicles." 2018 Second International Conference on Inventive Communication and Computational Technologies (ICICCT). IEEE, 2018.

[10] Javaid, Sabeen, et al. "Smart traffic management system using Internet of Things." 2018 20th International Conference on Advanced Communication Technology (ICACT). IEEE, 2018.

[11] Bhilawade, Vidya, and L. K. Ragha. "Intelligent Traffic Control System." International Journal of Scientific and Research Publications 8.2 (2018).

[12] Dutta, Arjun, et al. "Intelligent Traffic Control System: Towards Smart City." 2019 IEEE 10th Annual Information Technology, Electronics and Mobile Communication Conference (IEMCON). IEEE, 2019.

[13] Hashim, N. M. Z., et al. "Traffic light control system for emergency vehicles using radio frequency." IOSR Journal of Engineering (IOSRJEN) 3.7 (2013): 43-52.

[14] Jyothi, B. Naga. "Smart traffic control system using ATMEGA328 micro controller and Arduino software." 2016 International Conference on Signal Processing, Communication, Power and Embedded System (SCOPES). IEEE, 2016.

[15] Shetty, Mr Prashanth, Leo Lazar, and K. Vishnu Shekar. "Traffic Signal Control System With Ambulance Assistance." IOSR Journal of Electronics and Communication Engineering 12.4 (2017): 71-79.

[16] M.Srinivasan, K.Naveen, R.Srivatsan, J.Thanish, K.Ramkumar, Efficient Traffic Priority Control for Ambulance Clearance, International Journal Of Advanced Research In Science And Engineering Volume No. 7, Special Issue No. 2, February 2018.

[17] Djahel, Soufiene, et al. "Adaptive traffic management for secure and efficient emergency services in smart cities." 2013 IEEE International Conference on Pervasive Computing and Communications Workshops (PERCOM Workshops). IEEE, 2013.

[18] Shaikh, Farheena, and M. B. Chandak. "An approach towards traffic management system using density calculation and emergency vehicle alert." IOSR Journal of Computer Science (IOSR-JCE) (2014): 24-27.

[19] Nellore, Kapileswar, and Gerhard P. Hancke. "Traffic management for emergency vehicle priority based on visual sensing." Sensors 16.11 (2016): 1892.

[20] Munem, Asmaa S. Abdul, and Dr Muayad S. Croock. "Smart Traffic Light Control System for Emergency Ambulance." International Journal of Advanced Research in Computer Engineering and Technology (IJARCET) 5.8 (2016): 2247-2255.

[21] Ghazal, Bilal, et al. "Smart traffic light control system." 2016 third international conference on electrical, electronics, computer engineering and their applications (EECEA). IEEE, 2016.

[22] Arthi Sunderraj, Sabyasachi Routaray. "Intelligent Traffic Control System" International Journal Of Electrical, Electronics And Data Communication(2017). 
[23] Kanungo, Anurag, Ayush Sharma, and Chetan Singla. "Smart traffic lights switching and traffic density calculation using video processing." 2014 Recent Advances in Engineering and Computational Sciences (RAECS). IEEE, 2014.

[24] Jadhav, Prashant, et al. "Smart traffic control system using image processing." International Research Journal of Engineering and Technology (IRJET) 3.3 (2016): 2395-0056.

[25] Vani, R., et al. "Intelligent Traffic Control System with Priority to Emergency Vehicles."In IOP Conference Series: Materials Science and Engineering, vol. 455, no. 1, p. 012023. IOP Publishing, 2018.

[26] Sarath, S., and L. R. Deepthi. "Priority Based Real Time Smart Traffic Control System Using Dynamic Background." 2018 International Conference on Communication and Signal Processing (ICCSP). IEEE, 2018.

[27] Srinivasan, Varsha, et al. "Smart traffic control with ambulance detection." IOP Conf. Ser. Mater. Sci. Eng. Vol. 402. No. 1. 2018.

[28] Malik, Fehda, Munam Ali Shah, and Hasan Ali Khattak. "Intelligent Transport System: An Important Aspect of Emergency Management in Smart Cities." 2018 24th International Conference on Automation and Computing (ICAC). IEEE, 2018.

[29] Rath, Mamata. "Smart traffic management system for traffic control using automated mechanical and electronic devices." IOP Conference Series: Materials Science and Engineering. Vol. 377. No. 1. IOP Publishing, 2018.

[30] Roy, Shuvendu, and Md Sakif Rahman. "Emergency Vehicle Detection on Heavy Traffic Road from CCTV Footage Using Deep Convolutional Neural Network." 2019 International Conference on Electrical, Computer and Communication Engineering (ECCE). IEEE, 2019.

[31] Joshi, Nilakshi, et al. "Near real time vehicle tracking using GIS." 2015 International Conference on Technologies for Sustainable Development (ICTSD). IEEE, 2015.

[32] Dang, Dheeraj, Jitin Tanwar, and Sarfaraz Masood. "A smart traffic solution for High Priority Vehicles." 2015 1st international conference on next generation computing technologies (NGCT). IEEE, 2015.

[33] Khan, Ajmal, et al. "EVP-STC: Emergency vehicle priority and self-organizing traffic control at intersections using Internet-of-things platform." IEEE Access 6 (2018): 68242-68254.

[34] Iswarya, Gowram, H. Bharath, and V. Viharika Reddy. "Sound sensors to control traffic system for emergency vehicles." International Journal of Applied Engineering Research 13.7 (2018): 184-186.

[35] Kumar, G. Hemanth, and G. P. Ramesh. "Intelligent gateway for real time train tracking and railway crossing including emergency path using D2D communication." 2017 International Conference on Information Communication and Embedded Systems (ICICES). IEEE, 2017.

[36] Udawant, Omkar, et al. "Smart ambulance system using IoT." 2017 International Conference on Big Data, IoT and Data Science (BID). IEEE, 2017.

[37] George, Anita Acha, et al. "Golden aid an emergency ambulance system." 2017 International Conference on Networks \& Advances in Computational Technologies (NetACT). IEEE, 2017.

[38] Deshmukh, Sayalee, and S. B. Vanjale. "IOT Based Traffic Signal Control for Reducing Time Delay of an Emergency Vehicle Using GPS." 2018 Fourth International Conference on Computing Communication Control and Automation (ICCUBEA). IEEE, 2018.

[39] Karthikeyan, Sreehari, et al. "Smart and assistive driving headgear." 2018 3rd International Conference on Communication and Electronics Systems (ICCES). IEEE, 2018.

[40] Madisa, Mpho K., and Meera K. Joseph. "Android and Cloud Based Traffic Control System." 2018 International Conference on Advances in Big Data, Computing and Data Communication Systems (icABCD). IEEE, 2018. 
[41] Dhatrak, A. S., and S. T. Gandhe. "Automatic Traffic Signals in Smart Cities for Speedy Clearance of Emergency Vehicles." 2018 Fourth International Conference on Computing Communication Control and Automation (ICCUBEA). IEEE, 2018.

[42] Neha Sharma, Pooja Rampariya,Akshay Mahashabde, Aishwarya Mandale "Smart Traffic Signal Management for Emergency Vehicles Using FOG Computing", International Journal of Computer Science and Network, Volume 7, Issue 1, February 2018

[43] Kobayashi, Toru, et al. "Smart Ambulance Approach Alarm System Using Smartphone." IEICE Transactions on Information and Systems 102.9 (2019): 1689-1692.

[44] Almuraykhi, Khalid M., and Muhammad Akhlaq. "STLS: Smart Traffic Lights System for Emergency Response Vehicles." 2019 International Conference on Computer and Information Sciences (ICCIS). IEEE, 2019.

[45] Sangamesh, S. B., et al. "Advanced Traffic Signal Control System for Emergency Vehicles."

[46] Shyamala.S, R.Ranjith. Density based Traffic Control System Embedded with Patient Weightage Method, International Journal of Innovative Technology and Exploring Engineering (IJITEE) ISSN: 2278-3075, Volume-8, Issue-10S, August 2019

[47] Amaresh, A. M., et al. "Density Based Smart Traffic Control System for Congregating Traffic Information." 2019 International Conference on Intelligent Computing and Control Systems (ICCS). IEEE, 2019.

[48] Amer, Hayder M., et al. "Coalition game for emergency vehicles re-routing in smart cities." 2018 IEEE 10th Sensor Array and Multichannel Signal Processing Workshop (SAM). IEEE, 2018.

[49] Oeljeklaus, Malte, Frank Hoffmann, and Torsten Bertram. "A fast multi-task CNN for spatial understanding of traffic scenes." 2018 21st International Conference on Intelligent Transportation Systems (ITSC). IEEE, 2018.

[50] Priya, M. Deva, et al. "Intelligent navigation system for emergency vehicles." Proceedings of the 4th International Conference on Smart City Applications. 2019.

[51] Pulsiri, Nonthapat, et al. "Save Lives: A Review of Ambulance Technologies in Pre-Hospital Emergency Medical Services." 2019 Portland International Conference on Management of Engineering and Technology (PICMET). IEEE, 2019.

[52] Chinyere, Osigwe Uchenna, Oladipo Onaolapo Francisca, and Onibere Emmanuel Amano. "Design and simulation of an intelligent traffic control system." International journal of advances in engineering \& technology 1.5 (2011): 47. 